\section{Consumo alimentar entre crianças brasileiras com idade de 6 a 59 meses}

\author{
Food consumption Brazilian children by \\ 6 to 59 months of age
}

\author{
${ }^{1}$ Faculdade de Ciências \\ da Saúde, Universidade de \\ Brasília, Brasília, Brasil. \\ Correspondência \\ G. A. Bortolini \\ Departamento de Nutrição, \\ Faculdade de Ciências da \\ Saúde, Universidade de \\ Brasília. \\ Campus Universitário Darcy \\ Ribeiro, Brasília, DF \\ 70910-900, Brasil. \\ giselebortolini@hotmail.com
}

\section{Abstract}

The aim of this study was to assess food consumption in Brazilian children 6 to 59 months of age by region of the country and area of residence. This was a descriptive cross-sectional study of 4,322 children in the National Demographic and Health Survey (2006-2007). The data showed low daily consumption of leafy vegetables (12.7\%), vegetables (21.8\%), and meat (24.6\%) and high consumption (1-3 times a week) of soft drinks (40.5\%), fried foods (39.4\%), salty snacks (39.4\%), and sweets (37.8\%). Comparing the regions of Brazil, children in the South, Southeast, and Central-West consumed more rice, bread, potatoes, beans, greens, vegetables, and meat, but they also ate more foods not recommended for their age, like sweets and soft drinks (soda). Rural children showed lower consumption of foods recommended for their age and also those not recommended for their age, as compared to their urban counterparts. According to this study, food consumption in these young children fails to meet the recommendations for healthy eating in this age bracket.

Food Consumption; Health Promotion; Child Nutrition Sciences
Gisele Ane Bortolini 1

Muriel Bauermann Gubert 1

Leonor Maria Pacheco Santos 1

\section{Introdução}

O consumo alimentar inadequado nos primeiros anos de vida está relacionado à ocorrência de morbimortalidade em crianças, representada por doenças infecciosas, afecções respiratórias, cárie dental, desnutrição, excesso de peso e carências específicas de micronutrientes como ferro, zinco e vitamina A, entre outros 1,2,3,4,5,6,7,8. De acordo com o último inquérito nacional, 7,4\% das crianças brasileiras menores de cinco anos apresentavam excesso de peso, 1,6\% baixo peso e $6,8 \%$ baixa estatura. A anemia foi observada em $20,9 \%$ e a hipovitaminose A, em $17,4 \%$ das crianças ${ }^{9}$. Também se estima que mais de 200 milhões de crianças no mundo não atingem seu potencial de desenvolvimento, sendo a nutrição inadequada um dos fatores determinantes 10 .

O hábito alimentar é estabelecido nos primeiros anos de vida e repercute nas práticas alimentares e no estado de saúde e nutrição ao longo da vida 11,12,13,14. No Brasil, apesar dos avanços, a mediana de amamentação exclusiva observada para as crianças residentes nas capitais brasileiras e no Distrito Federal foi de 1,8 mês, e a de aleitamento materno foi de 11,2 meses, o que indica a introdução precoce de líquidos e alimentos 15 . Em outros estudos, já foram evidenciadas diferenças regionais na introdução precoce de alimentos. O chá foi oferecido com mais frequência nas capitais da Região Sul; leites e sucos foram mais ofertados nas capitais da Região Nordeste 
e Sudeste e o consumo precoce de mingau/papa foi mais elevado nas capitais da Região Nordeste 16. Também se observou elevado consumo de refrigerantes, café, bolachas e salgadinhos antes de um ano de idade 15.

Investir em ações integrais que garantam que as crianças se desenvolvam adequadamente é de suma importância para o crescimento de um país 17. Conhecer o perfil de saúde e nutrição da população permite subsidiar o melhor desenho de políticas públicas dirigidas às crianças. Dados sobre consumo alimentar dos brasileiros com até cinco anos de idade foram coletados, de forma inédita no país, pela Pesquisa Nacional de Demografia e Saúde (PNDS) ${ }^{9}$. Assim, o objetivo do presente estudo é apresentar a frequência de consumo de alimentos em crianças de 6 a 59 meses, de acordo com zona de residência (urbana ou rural) e região do país.

\section{Metodologia}

O presente trabalho utilizou dados secundários oriundos da Pesquisa Nacional de Demografia e Saúde (PNDS) de 2006/2007, que é parte do programa internacional Demographic Health Surveys (DHS). A PNDS se insere na quinta fase do DHS, realizado em escala mundial. No Brasil, é realizada com intervalos de dez anos e, no ano de 2006, entrou em sua terceira edição ${ }^{9,18}$.

A PNDS caracteriza-se por ser um estudo de corte transversal cujo público-alvo foram todas as mulheres em idade reprodutiva, definidas como aquelas com 15-49 anos de idade, que residiam nos domicílios selecionados, denominadas mulheres elegíveis para o estudo. Também foram investigados seus filhos menores de cinco anos de idade, ou seja, que nasceram a partir de janeiro de 2001. A pesquisa utilizou procedimentos complexos de amostragem envolvendo estratificação do conjunto de setores censitários do país, sorteio de conglomerados de setores dentro de estratos e sorteio de domicílios dentro dos setores. O universo em estudo é formado por domicílios particulares em setores comuns ou não especiais (inclusive favelas), selecionados em dez estratos amostrais que compõem uma combinação de todas as cinco grandes regiões geográficas brasileiras, além das áreas urbanas e rurais. Foram investigados 14.617 domicílios, $15.575 \mathrm{mu}$ lheres e 4.957 crianças menores de cinco anos, vivas no momento da entrevista 9,18. Trata-se de investigação que possui representatividade nacional para as cinco macrorregiões e para as zonas urbanas e rurais.

As informações foram obtidas mediante questionários aplicados face a face com as mu- lheres. O instrumento manteve a estrutura tradicional das pesquisas anteriores e as questões imprescindíveis para comparabilidade em âmbito nacional e internacional, porém incorporou novos temas. Os procedimentos de amostragem e coleta de dados estão descritos em detalhes em outras publicações 9,18 .

\section{Avaliação do consumo alimentar em crianças menores de cinco anos}

O questionário de frequência de consumo alimentar da criança foi aplicado a todos os responsáveis por menores de cinco anos, e os dados coletados dos questionários referentes às crianças de 6 a 59 meses foram utilizados no presente estudo ${ }^{19}$. Os alimentos avaliados neste trabalho, de acordo com a categoria de investigação do questionário, são: (a) arroz (arroz e macarrão); (b) pão; (c) batata (batata, mandioca, mandioquinha, cará, inhame, abóbora); (d) feijão (feijões e lentilha); (e) verduras de folhas; (f) legumes (tomate, cenoura, beterraba, berinjela, chuchu e abobrinha); (g) frutas; (h) carne (bovina e suína); (i) frango; (j) peixe; (k) suco natural de fruta; (l) doces; (m) biscoito (biscoito ou bolacha); (n) salgadinhos (salgadinhos industrializados de pacote); (o) refrigerantes (refrigerantes e sucos artificiais) e (p) iogurte (iogurtes).

As categorias de frequência de consumo analisadas neste estudo foram recodificadas para: (a) não ingeriu nos últimos sete dias; (b) ingeriu na frequência de 1-3 dias da semana; (c) ingeriu na frequência de 4-6 dias da semana e (d) ingeriu todos os dias. Foram excluídas da recodificação as respostas "não sabe" e "sem resposta", para as quais o percentual de respostas foi de aproximadamente $1 \%$ da amostra não expandida. O percentual variou de acordo com o alimento.

Os alimentos foram classificados em recomendados e não recomendados para essa faixa etária, segundo orientações do Ministério da Saúde 20,21. Os alimentos considerados recomendados são: arroz, pão, batata, feijão, verduras de folhas, legumes, frutas, carne, frango, peixe, suco natural de fruta e iogurte. Os alimentos considerados não recomendados são: doces, biscoito, salgadinhos e refrigerantes. O pão e o iogurte são recomendados para crianças a partir de 12 meses, as quais constituem a maior proporção na amostra; assim, optou-se por incluir esses alimentos na categoria de recomendados.

\section{Análises dos dados}

O programa utilizado para as análises dos dados foi o SPSS versão 16.0 (SPSS Inc., Chicago, Estados Unidos), as quais foram realizadas no módu- 
lo para amostras complexas. O plano de análise considerou o peso da amostra para crianças, o estrato e o conglomerado de residência. Todas as análises foram realizadas com amostra expandida, por isso os resultados são apresentados em percentuais. O banco de dados final foi composto por crianças de 6-59 meses, que moravam com a mãe, tendo permanecido no banco de dados apenas as crianças com resposta para o questionário de consumo. Os menores de seis meses foram excluídos em virtude da recomendação de serem exclusivamente amamentadas até essa idade. O banco contava com 4.957 crianças menores de 60 meses, das quais foram excluídas 140 que não residiam com suas mães e 495 que tinham de zero a cinco meses, resultando em tamanho amostral de 4.322 crianças. A amostra expandida corresponde a 13 milhões e $90 \mathrm{mil}$ crianças.

O consumo alimentar em crianças brasileiras foi investigado usando-se análise univariada apresentada em percentual e respectivo intervalo de confiança. Para a análise do consumo alimentar por região de residência e zona de residência, o teste de associação utilizado foi o qui-quadrado de Pearson, e a frequência das variáveis de desfechos foi apresentada sob a forma de percentuais e respectivos intervalos de $95 \%$ de confiança (IC95\%), sendo considerado nível de 5\% de significância.

\section{Aspectos éticos}

O estudo original foi aprovado pelo Conselho de Ética em Pesquisa do Centro de Referência e Treinamento DST/AIDS da Secretaria de Estado de Saúde de São Paulo.

\section{Resultados}

A maioria das mães (78\%) apresentava idade de 20-35 anos. Residiam em área rural 19,2\% (IC95\%: 16,7-21,9) das crianças e, em área urbana, 80,8\% (IC95\%: 78,1-83,3). A análise por sexo mostrou que $52,7 \%$ das crianças avaliadas eram do sexo masculino e $47,3 \%$, do sexo feminino. $\mathrm{O}$ percentual de crianças na idade de 6-23 meses correspondeu a $33 \%$, e $67 \%$ apresentavam idade de 24-59 meses.

A Tabela 1 apresenta a frequência de consumo semanal dos alimentos investigados em crianças brasileiras com idade entre 6-59 meses. É possível observar que $44,6 \%$ das crianças consumiram diariamente frutas; $12,7 \%$, verduras de folhas; $21,8 \%$, legumes; $24,6 \%$, carnes; $21,4 \%$, doces; 46,3\%, biscoitos; 8,5\%, salgadinhos; $22,1 \%$, refrigerantes. A frequência de crianças que consumiram feijão e arroz foi de $66,2 \%$ e $77,4 \%$ respectivamente. Destaca-se, ainda, que $53,2 \%$ das crianças não consumiram verduras de folhas, $25,3 \%$ não consumiram legumes e $11,5 \%$ não consumiram frutas em nenhuma ocasião, na semana de referência. A frequência de crianças que consumiram diariamente carne, frango e peixe somou, aproximadamente, $30 \%$ (Tabela 1).

\section{Consumo de arroz, pão, batata e feijão de acordo com a região de residência}

As maiores frequências de consumo diário de arroz, pão e batata foram observadas nas crianças residentes nas Regiões Centro-oeste, Sudeste e Sul, enquanto a menor frequência de consumo desses alimentos foi observada no Norte e Nordeste. Pode-se observar que $46,5 \%$ das crianças residentes na Região Norte e 44,3\% daquelas residentes na Região Nordeste não consumiram batata na última semana; esse valor é de aproximadamente $15 \%$ para as demais regiões. Também não consumiram pão, na última semana, 20,6\% das crianças residentes na Região Norte e 13,9\% das crianças da Região Nordeste; esses valores variaram de $7 \%$ a $10 \%$ nas demais regiões. Consumo diário de feijão foi observado com maior frequência nas crianças residentes no Centrooeste $(79 \%)$ e Sudeste $(78,5 \%)$ e com menor frequência nas residentes na Região Norte $(42,1 \%)$ (Tabela 2).

\section{Consumo de verduras de folhas, legumes, frutas, suco natural de fruta de acordo com a região de residência}

Os maiores percentuais de consumo diário de verduras de folhas foram observados nas crianças que residiam no Sul $(16,8 \%)$, Sudeste $(15,4 \%)$ e Centro-oeste (13,9\%); os de não consumo foram observados nas crianças residentes no Nordeste $(69,8 \%)$ e Norte $(59 \%)$. Os percentuais mais elevados de consumo diário de legumes foram observados nas crianças residentes no Sul (28,3\%), Sudeste $(23,3 \%)$ e Centro-oeste $(22,8 \%)$; os de não consumo foram observados nas crianças residentes no Norte $(15,7 \%)$ e Nordeste $(18,3 \%)$. $\mathrm{O}$ percentual de crianças que consumiram frutas diariamente foi semelhante nas Regiões Sul $(48,7 \%)$, Nordeste $(47,4 \%)$ e Sudeste $(46,6 \%)$; nas crianças do Centro-oeste, por sua vez, o consumo foi mais baixo. A maior frequência de crianças que não consumiram frutas na última semana foi observada na Região Norte $(21,7 \%)$. O consumo de suco natural de fruta foi mais elevado nas crianças do Nordeste $(41,8 \%)$ e Sudeste $(32,5 \%)$ (Tabela 2). 
Tabela 1

Consumo alimentar semanal em crianças com idade de 6-59 meses. Brasil, 2006/2007 *

\begin{tabular}{|c|c|c|c|c|c|c|c|c|}
\hline \multirow[t]{2}{*}{ Categoria de alimentos } & \multicolumn{2}{|c|}{ Não consumiu } & \multicolumn{2}{|c|}{$1-3$ vezes } & \multicolumn{2}{|c|}{$4-6$ vezes } & \multicolumn{2}{|c|}{ Todos os dias } \\
\hline & $\%$ & IC95\% & $\%$ & IC95\% & $\%$ & IC95\% & $\%$ & IC95\% \\
\hline Arroz ** & 3,2 & $2,4-4,1$ & 12,8 & $10,9-14,9$ & 6,7 & $5,4-8,4$ & 77,4 & $75,0-79,5$ \\
\hline Pão & 11,0 & $9,3-13,0$ & 28,7 & $26,2-31,4$ & 8,2 & $6,6-10,2$ & 52,0 & $49,2-54,8$ \\
\hline Batata *** & 25,3 & $22,7-28,1$ & 49,7 & $46,1-53,2$ & 9,6 & $8,1-11,3$ & 15,4 & $13,4-17,7$ \\
\hline Feijão \# & 7,9 & $6,7-9,3$ & 18,2 & $16,1-20,5$ & 7,7 & $6,4-9,2$ & 66,2 & $63,5-68,8$ \\
\hline Verduras de folhas & 53,2 & $50,3-56,2$ & 28,6 & $26,0-31,2$ & 5,5 & $4,4-6,9$ & 12,7 & $11,0-14,6$ \\
\hline Legumes \#\# & 25,3 & $22,9-27,9$ & 39,0 & $35,8-42,2$ & 13,9 & $12,1-15,9$ & 21,8 & $19,3-24,6$ \\
\hline Frutas & 11,5 & $9,9-13,3$ & 30,4 & $27,8-33,1$ & 13,5 & $11,6-15,5$ & 44,6 & $41,5-47,8$ \\
\hline Carne \#\#\# & 17,2 & $14,9-19,6$ & 44,1 & $40,9-47,4$ & 14,1 & $12,4-16,1$ & 24,6 & $21,9-27,5$ \\
\hline Frango & 21,9 & $19,1-24,9$ & 58,4 & $55,3-61,5$ & 13,6 & $11,7-15,6$ & 6,1 & $4,8-7,9$ \\
\hline Peixe & 58,0 & $55,0-61,0$ & 36,5 & $33,6-39,6$ & 3,9 & $3,1-4,9$ & 1,5 & $1,0-2,3$ \\
\hline Suco natural de fruta & 24,1 & $21,6-27,3$ & 29,8 & $27,3-32,4$ & 13,4 & $11,7-15,3$ & 32,5 & $29,7-35,4$ \\
\hline Alimentos fritos & 49,1 & $45,7-52,5$ & 39,4 & $36,1-42,8$ & 6,1 & $4,9-7,5$ & 5,5 & $4,2-7,1$ \\
\hline Doces & 28,6 & $26,0-31,3$ & 37,8 & $34,7-41,1$ & 12,2 & $10,4-14,3$ & 21,4 & $18,7-24,3$ \\
\hline Biscoitos § & 8,7 & $7,3-10,4$ & 26,8 & $23,6-30,3$ & 18,2 & $15,9-20,7$ & 46,3 & $42,4-50,3$ \\
\hline Salgadinhos $\S \S$ & 44,7 & $41,8-47,7$ & 39,4 & $36,6-42,2$ & 7,4 & $5,9-9,2$ & 8,5 & $6,9-10,4$ \\
\hline Refrigerante §§§ & 26,3 & $23,9-28,8$ & 40,5 & $37,1-44,0$ & 11,1 & $9,3-13,2$ & 22,1 & $19,5-24,8$ \\
\hline logurte & 24,0 & $21,9-26,9$ & 34,9 & $32,5-37,4$ & 15,1 & $12,3-18,3$ & 25,7 & $23,1-28,4$ \\
\hline
\end{tabular}

IC95\%: intervalo de 95\% de confiança.

* $\mathrm{n}$ amostral sem expansão $=4.322$;

** Arroz e macarrão;

*** Batata, mandioca, mandioquinha (batata-baroa), cará, inhame, abóbora;

\# Feijões e lentilha;

\#\# Tomate, cenoura, beterraba, berinjela, chuchu e abobrinha;

\#\#\# Carne bovina e suína;

$\S$ Biscoitos e bolachas;

$\S \S$ Salgadinhos de pacote;

$\S \S$ Refrigerantes e sucos artificiais.

Consumo de carne, frango, peixe e iogurte de acordo com a região de residência

Consumiram carne bovina ou de porco, diariamente, $38,3 \%$ das crianças residentes na Região Sul, 34,9\% das residentes no Centro-oeste e $25,2 \%$ das crianças do Sudeste. Aproximadamente, $20 \%$ das residentes no Norte e Nordeste não consumiram carne na semana de referência. O percentual das que consumiram frango diariamente foi de $10,7 \%$ na Região Sul e de $7,7 \%$ na Região Nordeste. Os percentuais mais elevados de consumo diário de peixe foram observados nas crianças residentes no Norte $(7 \%)$ e Nordeste $(2,7 \%)$. O consumo diário de iogurte foi mais elevado nas crianças do Sul $(34,2 \%)$ e Sudeste (31,5\%) (Tabela 2).
Consumo de alimentos fritos, doces, biscoitos, salgadinhos e refrigerantes de acordo com a região de residência

O consumo de frituras na frequência de uma a três vezes na semana, entre as crianças brasileiras, foi de $44,4 \%$ e $44 \%$ no Sudeste e no Sul, respectivamente. A maior frequência das que consumiram doces diariamente foi observada na Região Sul $(37,2 \%)$, seguida da Região Sudeste (23\%). Aproximadamente, $50 \%$ das crianças de todas as regiões do Brasil, com exceção da Região Norte, cuja frequência é de 28,2\%, consomem biscoitos ou bolachas diariamente. O consumo diário de salgadinhos foi mais elevado entre as residentes no Nordeste $(10,7 \%)$. As maiores frequências de consumo diário de refrigerantes foram observadas nas residentes na Região Sul $(36,4 \%)$ e, em seguida, na Região Sudeste (29,3\%) (Tabela 2$)$. 
Consumo alimentar semanal em crianças com idade de 6-59 meses segundo a região de residência. Brasil, 2006/2007 *.

\begin{tabular}{|c|c|c|c|c|c|c|c|c|c|}
\hline \multirow[t]{2}{*}{ Categoria de alimentos } & \multicolumn{2}{|c|}{ Não consumiu } & \multicolumn{2}{|c|}{$1-3$ vezes } & \multicolumn{2}{|c|}{ 4-6 vezes } & \multicolumn{2}{|c|}{ Todos os dias } & \multirow[t]{2}{*}{ Valor de $\mathrm{p}$} \\
\hline & $\%$ & IC95\% & $\%$ & IC95\% & $\%$ & IC95\% & $\%$ & IC95\% & \\
\hline Arroz ** & & & & & & & & & $<0,001$ \\
\hline Norte & 6,0 & $4,3-8,5$ & 19,4 & $13,7-26,8$ & 7,8 & $5,3-11,2$ & 66,8 & $60,1-72,8$ & \\
\hline Nordeste & 4,1 & $2,6-6,5$ & 19,8 & $15,5-24,9$ & 10,1 & $7,2-13,9$ & 66,0 & $61,3-70,5$ & \\
\hline Sudeste & 2,6 & $1,5-4,3$ & 7,6 & $5,2-11,0$ & 4,7 & $2,6-8,2$ & 85,1 & $81,2-88,4$ & \\
\hline Sul & 1,2 & $0,6-2,6$ & 12,3 & $9,1-16,5$ & 6,6 & $4,7-9,3$ & 79,9 & $75,4-83,6$ & \\
\hline Centro-oeste & 2,4 & $1,3-4,5$ & 9,3 & $6,6-13,0$ & 5,2 & $3,6-7,6$ & 83,1 & $78,7-86,7$ & \\
\hline Pão & & & & & & & & & $<0,001$ \\
\hline Norte & 20,6 & $15,7-26,5$ & 23,2 & $19,0-28,0$ & 8,2 & $5,8-11,3$ & 48,0 & $41,9-54,2$ & \\
\hline Nordeste & 13,9 & $10,4-18,4$ & 33,9 & $29,3-38,9$ & 8,3 & $6,1-11,1$ & 43,8 & $37,5-50,4$ & \\
\hline Sudeste & 7,9 & $5,4-11,4$ & 30,7 & $26,0-35,8$ & 8,5 & $5,5-12,9$ & 52,9 & $48,3-57,5$ & \\
\hline Sul & 7,6 & $5,3-10,8$ & 19,5 & $15,9-23,7$ & 5,3 & $3,4-8,4$ & 67,6 & $63,2-71,6$ & \\
\hline Centro-oeste & 10,9 & $7,8-14,9$ & 23,0 & $19,2-27,3$ & 11,5 & $8,1-16,2$ & 54,6 & $48,1-60,9$ & \\
\hline 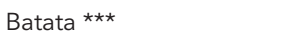 & & & & & & & & & $<0,001$ \\
\hline Norte & 46,5 & $40,6-52,5$ & 33,3 & $28,8-38,1$ & 10,4 & $7,7-13,9$ & 9,8 & $7,2-13,1$ & \\
\hline Nordeste & 44,3 & $38,8-50,0$ & 40,6 & $35,0-46,4$ & 6,1 & $4,2-8,6$ & 9,0 & $6,3-12,7$ & \\
\hline Sudeste & 14,1 & $10,4-18,8$ & 56,9 & $50,0-63,5$ & 9,0 & $6,4-12,5$ & 20,0 & $15,8-25,0$ & \\
\hline Sul & 12,8 & $9,8-16,5$ & 55,2 & $50,0-60,3$ & 15,4 & $12,0-19,6$ & 16,6 & $13,2-20,6$ & \\
\hline Centro-oeste & 14,5 & $11,4-18,3$ & 53,5 & $48,5-58,5$ & 14,2 & $10,9-18,3$ & 17,7 & $14,0-22,2$ & \\
\hline Feijão \# & & & & & & & & & $<0,001$ \\
\hline Norte & 18,7 & $15,0-23,2$ & 29,9 & $25,1-35,1$ & 9,3 & $6,8-12,5$ & 42,1 & $35,0-49,5$ & \\
\hline Nordeste & 11,4 & $8,6-14,9$ & 25,7 & $21,9-29,9$ & 9,5 & $7,0-12,8$ & 53,4 & $47,8-58,9$ & \\
\hline Sudeste & 3,6 & $2,2-5,9$ & 12,3 & $8,7-17,2$ & 5,5 & $3,6-8,2$ & 78,5 & $74,0-82,4$ & \\
\hline Sul & 7,6 & $5,1-11,0$ & 18,5 & $14,6-23,0$ & 10,6 & $7,9-14,1$ & 63,4 & $57,9-68,6$ & \\
\hline Centro-oeste & 5,3 & $3,3-8,5$ & 9,0 & $6,6-12,2$ & 6,7 & $4,8-9,3$ & 79,0 & $74,7-82,8$ & \\
\hline Verduras de folhas & & & & & & & & & $<0,001$ \\
\hline Norte & 59,0 & $54,0-63,8$ & 24,7 & $20,6-29,4$ & 6,7 & $4,5-10,0$ & 9,6 & $6,8-13,3$ & \\
\hline Nordeste & 69,8 & $64,0-75,0$ & 20,6 & $16,6-25,3$ & 2,5 & $1,4-4,4$ & 7,1 & $5,0-9,9$ & \\
\hline Sudeste & 44,8 & $39,7-50,0$ & 34,0 & $29,5-38,9$ & 5,8 & $3,8-8,7$ & 15,4 & $12,0-19,6$ & \\
\hline Sul & 48,0 & $43,1-52,9$ & 28,8 & $24,4-33,6$ & 6,5 & $4,3-9,7$ & 16,8 & $13,7-20,3$ & \\
\hline Centro-oeste & 45,0 & $39,3-50,8$ & 30,2 & $25,8-35,0$ & 11,0 & $7,5-15,7$ & 13,9 & $10,0-18,1$ & \\
\hline Legumes \#\# & & & & & & & & & $<0,001$ \\
\hline Norte & 43,9 & $38,6-49,2$ & 27,9 & $23,6-32,7$ & 12,5 & $9,8-15,7$ & 15,7 & $12,4-19,8$ & \\
\hline Nordeste & 33,3 & $29,1-37,8$ & 36,0 & $30,9-41,6$ & 12,3 & $9,2-16,3$ & 18,3 & $14,7-22,5$ & \\
\hline Sudeste & 18,2 & $14,1-23,1$ & 43,6 & $37,7-49,7$ & 14,6 & $11,6-19,0$ & 23,3 & $18,2-29,4$ & \\
\hline Sul & 22,6 & $18,3-27,6$ & 37,6 & $32,2-43,3$ & 11,5 & $8,9-14,9$ & 28,3 & $24,0-33,0$ & \\
\hline Centro-oeste & 16,8 & $13,6-20,7$ & 40,7 & $35,1-46,6$ & 19,7 & $16,1-23,8$ & 22,8 & $18,8-27,4$ & \\
\hline Frutas & & & & & & & & & $<0,001$ \\
\hline Norte & 21,7 & $18,1-25,8$ & 32,9 & $28,4-37,7$ & 16,9 & $13,4-21,0$ & 28,6 & $23,8-33,9$ & \\
\hline Nordeste & 10,4 & $7,6-14,1$ & 27,4 & $22,6-32,8$ & 14,8 & $11,5-18,8$ & 47,4 & $41,3-53,6$ & \\
\hline Sudeste & 10,2 & $7,5-13,8$ & 31,6 & $27,1-36,5$ & 11,6 & $8,4-15,8$ & 46,6 & $40,7-52,6$ & \\
\hline Sul & 10,4 & $7,5-14,3$ & 29,8 & $24,8-35,2$ & 11,2 & $8,6-14,4$ & 48,7 & $43,1-54,3$ & \\
\hline Centro-oeste & 10,9 & $8,4-14,0$ & 31,8 & $27,4-36,5$ & 18,5 & $14,8-22,8$ & 38,8 & $33,8-44,1$ & \\
\hline
\end{tabular}

(continua) 
Tabela 2 (continuação)

\begin{tabular}{|c|c|c|c|c|c|c|c|c|c|}
\hline \multirow[t]{2}{*}{ Categoria de alimentos } & \multicolumn{2}{|c|}{ Não consumiu } & \multicolumn{2}{|c|}{$1-3$ vezes } & \multicolumn{2}{|c|}{ 4-6 vezes } & \multicolumn{2}{|c|}{ Todos os dias } & \multirow[t]{2}{*}{ Valor de $p$} \\
\hline & $\%$ & IC95\% & $\%$ & IC95\% & $\%$ & IC95\% & $\%$ & IC95\% & \\
\hline Carnes \#\#\# & & & & & & & & & $<0,001$ \\
\hline Norte & 21,9 & $16,9-27,8$ & 39,9 & $34,9-45,0$ & 20,7 & $16,5-25,6$ & 17,6 & $13,7-22,2$ & \\
\hline Nordeste & 22,0 & $18,2-26,3$ & 46,5 & $42,3-50,7$ & 14,9 & $11,5-19,1$ & 16,6 & $12,9-21,1$ & \\
\hline Sudeste & 15,3 & $11,3-20,3$ & 48,8 & $42,4-55,3$ & 10,7 & $8,0-14,1$ & 25,2 & $19,9-31,3$ & \\
\hline Sul & 13,4 & $10,0-17,6$ & 32,6 & $27,8-37,8$ & 15,7 & $12,2-20,0$ & 38,3 & $32,9-44,0$ & \\
\hline Centro-oeste & 10,9 & $8,2-14,4$ & 35,7 & $30,9-40,7$ & 18,6 & $15,5-22,1$ & 34,9 & $30,3-39,7$ & \\
\hline Frango & & & & & & & & & 0,030 \\
\hline Norte & 29,8 & $25,5-34,5$ & 55,0 & $50,2-59,6$ & 12,6 & $9,3-16,9$ & 2,7 & $1,3-5,4$ & \\
\hline Nordeste & 20,0 & $16,4-24,2$ & 57,6 & $52,1-63,0$ & 14,7 & $11,5-18,5$ & 7,7 & $4,8-12,0$ & \\
\hline Sudeste & 22,0 & $16,5-28,8$ & 59,7 & $53,4-65,7$ & 13,1 & $9,8-17,3$ & 5,2 & $3,2-8,2$ & \\
\hline Sul & 16,9 & $13,6-20,7$ & 59,4 & $54,0-64,6$ & 13,0 & $10,1-16,5$ & 10,7 & $7,2-15,5$ & \\
\hline Centro-oeste & 25,3 & $21,1-30,0$ & 57,0 & $51,9-62,1$ & 14,6 & $11,6-18,3$ & 3,1 & $1,9-5,0$ & \\
\hline Peixe & & & & & & & & & $<0,001$ \\
\hline Norte & 41,3 & $36,1-46,6$ & 41,3 & $37,0-45,8$ & 10,5 & $7,4-14,7$ & 7,0 & $4,1-11,5$ & \\
\hline Nordeste & 50,7 & $45,7-55,7$ & 41,3 & $36,6-46,1$ & 5,4 & $3,8-7,6$ & 2,7 & $1,4-5,0$ & \\
\hline Sudeste & 61,8 & $55,6-67,7$ & 35,9 & $30,1-42,2$ & 2,1 & $1,0-4,2$ & 0,1 & $0,0-0,6$ & \\
\hline Sul & 67,0 & $62,4-71,4$ & 30,7 & $26,5-35,3$ & 1,9 & $1,0-3,5$ & 0,3 & $0,1-0,8$ & \\
\hline Centro-oeste & 69,1 & $64,1-73,8$ & 27,2 & $23,0-31,7$ & 3,5 & $2,2-5,5$ & 0,2 & $0,1-0,8$ & \\
\hline Suco natural de fruta & & & & & & & & & $<0,001$ \\
\hline Norte & 28,3 & $23,8-33,3$ & 34,2 & $30,0-38,8$ & 16,6 & $12,8-21,3$ & 20,9 & $17,2-25,1$ & \\
\hline Nordeste & 14,5 & $10,8-19,3$ & 25,6 & $21,2-30,6$ & 18,0 & $14,5-22,2$ & 41,8 & $36,8-46,9$ & \\
\hline Sudeste & 25,9 & $20,8-31,8$ & 31,3 & $26,5-36,4$ & 10,3 & $7,6-13,8$ & 32,5 & $27,3-38,2$ & \\
\hline Sul & 36,5 & $31,5-41,8$ & 27,6 & $23,5-32,1$ & 10,7 & $8,1-14,2$ & 25,2 & $21,1-29,7$ & \\
\hline Centro-oeste & 24,1 & $19,4-29,4$ & 34,0 & $29,4-38,9$ & 14,0 & $10,9-17,8$ & 27,9 & $23,8-32,4$ & \\
\hline Alimentos fritos & & & & & & & & & 0,001 \\
\hline Norte & 54,7 & $49,7-59,6$ & 34,8 & $30,2-39,7$ & 6,6 & $4,8-9,2$ & 3,9 & $2,6-5,8$ & \\
\hline Nordeste & 59,2 & $54,1-64,2$ & 30,8 & $25,5-36,6$ & 4,7 & $3,0-7,2$ & 5,3 & $3,1-8,9$ & \\
\hline Sudeste & 42,3 & $35,4-49,5$ & 44,4 & $37,8-51,2$ & 6,8 & $4,6-10,0$ & 6,5 & $4,2-9,7$ & \\
\hline Sul & 44,5 & $39,2-49,9$ & 44,0 & $38,8-49,2$ & 5,9 & $3,9-8,7$ & 5,7 & $3,1-10,3$ & \\
\hline Centro-oeste & 51,7 & $46,7-56,7$ & 39,7 & $34,8-44,8$ & 6,5 & $4,6-9,1$ & 2,1 & $1,2-3,6$ & \\
\hline Doces & & & & & & & & & $<0,001$ \\
\hline Norte & 38,2 & $33,2-43,4$ & 37,8 & $33,9-42,0$ & 12,6 & $9,9-15,8$ & 11,4 & $8,6-15,0$ & \\
\hline Nordeste & 32,1 & $27,7-36,9$ & 40,2 & $36,1-44,5$ & 12,9 & $9,8-16,8$ & 14,8 & $10,8-19,9$ & \\
\hline Sudeste & 27,1 & $22,3-32,6$ & 38,1 & $31,6-45,1$ & 11,5 & $8,2-15,8$ & 23,3 & $18,0-29,6$ & \\
\hline Sul & 19,1 & $15,8-22,9$ & 31,1 & $27,0-35,6$ & 12,5 & $9,6-16,2$ & 37,2 & $32,5-42,3$ & \\
\hline Centro-oeste & 28,0 & $23,7-32,7$ & 39,2 & $35,0-43,5$ & 12,6 & $10,0-15,9$ & 20,2 & $16,6-24,4$ & \\
\hline Biscoitos § & & & & & & & & & 0,002 \\
\hline Norte & 17,4 & $14,2-21,2$ & 34,2 & $29,2-39,5$ & 20,2 & $16,1-25,1$ & 28,2 & $23,5-33,4$ & \\
\hline Nordeste & 7,8 & $5,5-10,9$ & 22,9 & $19,4-26,7$ & 20,0 & $16,2-24,5$ & 49,3 & $43,5-55,1$ & \\
\hline Sudeste & 6,8 & $4,4-10,2$ & 27,9 & $21,3-35,6$ & 16,8 & $12,6-22,1$ & 48,5 & $40,2-56,9$ & \\
\hline Sul & 9,5 & $7,3-12,4$ & 25,5 & $21,5-29,9$ & 16,4 & $12,5-21,1$ & 48,6 & $43,3-53,9$ & \\
\hline Centro-oeste & 9,6 & $7,2-12,7$ & 26,8 & $22,3-31,9$ & 19,3 & $16,0-23,0$ & 44,3 & $39,3-49,5$ & \\
\hline Salgadinhos §§ & & & & & & & & & 0,025 \\
\hline Norte & 55,5 & $50,0-60,8$ & 35,4 & $30,4-40,7$ & 3,6 & $2,4-5,3$ & 5,5 & $3,6-8,4$ & \\
\hline Nordeste & 37,7 & $33,2-42,4$ & 42,1 & $37,5-46,8$ & 9,5 & $7,1-12,7$ & 10,7 & $8,4-13,5$ & \\
\hline Sudeste & 47,4 & $41,6-53,3$ & 37,9 & $32,5-43,5$ & 6,7 & $4,0-11,0$ & 8,0 & $5,1-12,3$ & \\
\hline Sul & 42,4 & $37,1-47,9$ & 41,8 & $36,6-47,1$ & 7,5 & $5,4-10,5$ & 8,2 & $9,7-11,8$ & \\
\hline Centro-oeste & 43,6 & $38,2-49,1$ & 39,9 & $34,7-45,5$ & 8,5 & $6,3-11,5$ & 8,0 & $5,5-11,5$ & \\
\hline
\end{tabular}

(continua) 
Tabela 2 (continuação)

\begin{tabular}{|c|c|c|c|c|c|c|c|c|c|}
\hline \multirow[t]{2}{*}{ Categoria de alimentos } & \multicolumn{2}{|c|}{ Não consumiu } & \multicolumn{2}{|c|}{$1-3$ vezes } & \multicolumn{2}{|c|}{$4-6$ vezes } & \multicolumn{2}{|c|}{ Todos os dias } & \multirow[t]{2}{*}{ Valor de $p$} \\
\hline & $\%$ & IC95\% & $\%$ & IC95\% & $\%$ & IC95\% & $\%$ & IC95\% & \\
\hline Refrigerantes §§§ & & & & & & & & & $<0,001$ \\
\hline Norte & 37,4 & $31,9-43,3$ & 37,7 & $33,3-42,2$ & 12,1 & $9,4-15,4$ & 12,8 & $9,6-17,0$ & \\
\hline Nordeste & 34,3 & $30,0-38,8$ & 45,3 & $39,9-50,7$ & 11,5 & $7,6-17,1$ & 9,0 & $6,4-12,4$ & \\
\hline Sudeste & 20,6 & $16,6-25,2$ & 40,3 & $33,4-47,6$ & 9,9 & $7,1-13,5$ & 29,3 & $23,9-35,3$ & \\
\hline Sul & 20,1 & $16,4-24,4$ & 32,0 & $27,7-36,7$ & 11,5 & $8,6-15,3$ & 36,4 & $31,7-41,3$ & \\
\hline Centro-oeste & 25,5 & $21,2-30,4$ & 43,5 & $38,8-48,4$ & 14,9 & $11,5-19,2$ & 16,1 & $12,8-19,9$ & \\
\hline logurte & & & & & & & & & $<0,001$ \\
\hline Norte & 44,2 & $38,5-50,1$ & 32,1 & $27,5-37,1$ & 12,3 & $9,3-16,0$ & 11,4 & $8,6-15,0$ & \\
\hline Nordeste & 30,6 & $25,9-35,8$ & 35,4 & $31,0-40,1$ & 15,9 & $12,5-19,9$ & 18,1 & $13,9-23,2$ & \\
\hline Sudeste & 16,1 & $12,3-20,7$ & 36,6 & $32,2-41,4$ & 15,8 & $10,4-23,3$ & 31,5 & $26,4-37,0$ & \\
\hline Sul & 23,1 & $19,0-27,8$ & 29,7 & $25,0-34,9$ & 12,9 & $9,8-16,8$ & 34,2 & $29,0-39,8$ & \\
\hline Centro-oeste & 23,7 & $20,0-27,8$ & 36,6 & $32,0-41,4$ & 15,7 & $12,5-19,6$ & 24,0 & $20,4-28,0$ & \\
\hline
\end{tabular}

IC95\%: intervalo de 95\% de confiança.

* $\mathrm{n}$ amostral sem expansão $=4.322$;

** Arroz e macarrão;

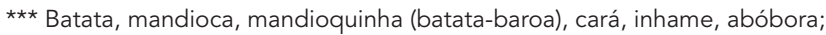

\# Feijões e lentilha;

\#\# Tomate, cenoura, beterraba, berinjela, chuchu e abobrinha;

\#\#\# Carne bovina e suína;

$\S$ Biscoitos e bolachas;

$\S \S$ Salgadinhos de pacote;

$\S \S \S$ Refrigerantes e sucos artificiais.

Síntese do consumo por região de residência

A Figura 1 apresenta a diferença, em pontos percentuais do consumo diário dos alimentos, observada para as crianças por região de residência, comparada à frequência nacional (Brasil). Dessa forma, é possível visualizar-se no gráfico o quanto o consumo das crianças de cada região se distancia positivamente ou negativamente do consumo diário nacional, sumarizando os dados das Tabelas 1 e 2 . As maiores variações de consumo foram observadas para feijão, refrigerantes, doces e pão. As menores variações, em relação ao consumo nacional, foram observadas para os alimentos frios e salgadinhos.

As crianças residentes na Região Norte consumiram com menor frequência todos os alimentos investigados, se comparados esses dados ao que se observa para todas as crianças do Brasil, com exceção do peixe. De forma contrária, as crianças residentes nas Regiões Sul e Sudeste apresentaram as maiores frequências de consumo para a maioria dos alimentos investigados. As crianças residentes no Centro-oeste consumiram, com maior frequência, a maioria dos alimentos recomendados e, com menor frequência, todos os alimentos não recomendados, em com- paração com a frequência para o Brasil. Por sua vez, as crianças residentes na Região Nordeste consumiram com menor frequência a maioria dos alimentos recomendados e não recomendados, no entanto consumiram em maior frequência frutas, frango, peixe, suco natural, biscoitos e salgadinhos, quando comparadas às crianças das outras regiões brasileiras.

\section{Consumo alimentar de acordo com a zona de residência: urbano ou rural}

É possível observar que a frequência de crianças que consumiram feijão, arroz, suco natural de fruta, frango, frituras e salgadinhos não variou com o local da residência: urbana e rural. Contudo, a frequência de crianças que consumiram diariamente pão, batata, verduras de folhas, legumes, frutas, iogurte, carnes, doces, biscoitos e refrigerante foi mais elevada nas crianças que residem na zona urbana. O consumo diário de peixe foi mais frequente nas crianças residentes na zona rural (Tabela 3). 
Figura 1

Diferença, em pontos percentuais, do consumo diário dos alimentos, observada para as crianças por região de residência, comparada à frequência nacional. Brasil, 2006/2007 *

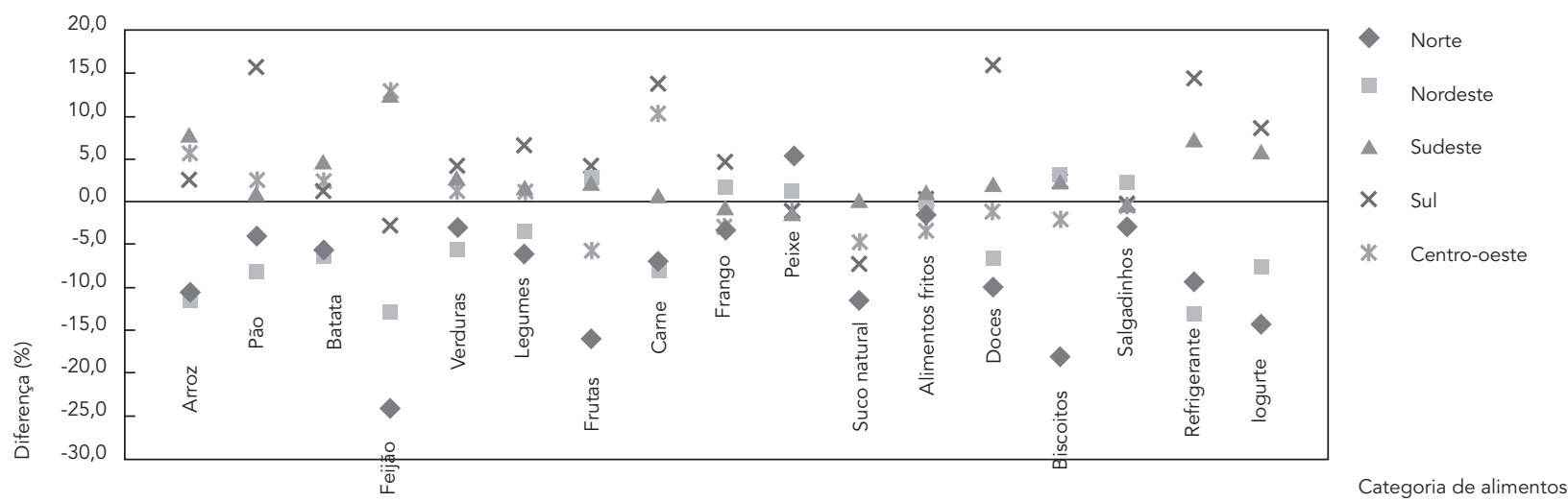

* $\mathrm{n}$ amostral sem expansão $=4.322$.

Tabela 3

Consumo alimentar semanal em crianças com idade de 6-59 meses segundo a zona de residência. Brasil, 2006/2007 *.

\begin{tabular}{|c|c|c|c|c|c|c|c|c|c|}
\hline \multirow[t]{2}{*}{ Categoria de alimentos } & \multicolumn{2}{|c|}{ Não consumiu } & \multicolumn{2}{|c|}{$1-3$ vezes } & \multicolumn{2}{|c|}{$4-6$ vezes } & \multicolumn{2}{|c|}{ Todos os dias } & \multirow[t]{2}{*}{ Valor de $p$} \\
\hline & $\%$ & IC95\% & $\%$ & IC95\% & $\%$ & IC95\% & $\%$ & IC95\% & \\
\hline Arroz ** & & & & & & & & & 0,133 \\
\hline Urbano & 3,1 & $2,3-4,1$ & 12,2 & $10,1-14,6$ & 6,1 & $4,7-8,0$ & 78,6 & $75,9-81,0$ & \\
\hline Rural & 3,4 & $1,9-6,0$ & 15,3 & $11,1-20,7$ & 9,1 & $6,2-13,1$ & 72,2 & $67,4-76,4$ & \\
\hline Pão & & & & & & & & & $<0,001$ \\
\hline Urbano & 9,2 & $7,4-11,3$ & 28,3 & $25,3-31,4$ & 8,6 & $6,7-11,0$ & 54,0 & $50,9-57,0$ & \\
\hline Rural & 18,7 & $14,1-24,5$ & 30,8 & $26,1-35,9$ & 6,6 & $4,6-9,4$ & 43,9 & $36,9-51,1$ & \\
\hline Batata *** & & & & & & & & & $<0,001$ \\
\hline Urbano & 22,4 & $19,7-25,5$ & 51,1 & $47,0-55,3$ & 9,6 & $7,9-11,6$ & 16,9 & $14,4-19,6$ & \\
\hline Rural & 37,2 & $31,6-43,3$ & 43,6 & $37,8-49,6$ & 9,8 & $7,3-13,0$ & 9,4 & $6,9-12,7$ & \\
\hline Feijão \# & & & & & & & & & 0,063 \\
\hline Urbano & 7,3 & $6,0-8,8$ & 17,5 & $15,1-20,2$ & 7,9 & $6,4-9,7$ & 67,3 & $64,3-70,2$ & \\
\hline Rural & 10,4 & $7,5-14,4$ & 21,2 & $17,9-25,0$ & 6,8 & $4,8-9,6$ & 61,5 & $55,6-67,1$ & \\
\hline Verduras de folhas & & & & & & & & & $<0,001$ \\
\hline Urbano & 50,7 & $47,4-54,0$ & 29,7 & $26,8-32,7$ & 5,9 & $4,6-7,6$ & 13,6 & $11,6-16,0$ & \\
\hline Rural & 63,8 & $57,7-69,5$ & 23,8 & $19,4-28,9$ & 3,8 & $2,8-5,3$ & 8,5 & $6,3-11,5$ & \\
\hline Legumes \#\# & & & & & & & & & $<0,001$ \\
\hline Urbano & 22,4 & $19,7-25,3$ & 39,6 & $36,0-43,3$ & 14,1 & $12,1-16,5$ & 24,0 & $20,8-27,4$ & \\
\hline Rural & 37,6 & $33,0-42,4$ & 36,6 & $30,6-43,1$ & 12,9 & $9,6-17,3$ & 12,9 & $10,1-16,3$ & \\
\hline Frutas & & & & & & & & & 0,010 \\
\hline Urbano & 10,2 & $8,5-12,3$ & 30,0 & $27,1-33,1$ & 13,6 & $11,5-16,0$ & 46,2 & $42,6-49,9$ & \\
\hline Rural & 17,0 & $13,5-21,2$ & 31,9 & $26,8-37,6$ & 13,0 & $9,9-17,0$ & 38,0 & $31,3-45,2$ & \\
\hline
\end{tabular}

(continua) 
Tabela 3 (continuação)

\begin{tabular}{|c|c|c|c|c|c|c|c|c|c|}
\hline \multirow[t]{2}{*}{ Categoria de alimentos } & \multicolumn{2}{|c|}{ Não consumiu } & \multicolumn{2}{|c|}{$1-3$ vezes } & \multicolumn{2}{|c|}{$4-6$ vezes } & \multicolumn{2}{|c|}{ Todos os dias } & \multirow[t]{2}{*}{ Valor de $p$} \\
\hline & $\%$ & IC95\% & $\%$ & IC95\% & $\%$ & IC95\% & $\%$ & IC95\% & \\
\hline Carne \#\#\# & & & & & & & & & 0,039 \\
\hline Urbano & 16,1 & $13,6-18,9$ & 44,1 & $40,3-48,1$ & 14,0 & $12,0-16,2$ & 25,8 & $22,6-29,4$ & \\
\hline Rural & 21,7 & $17,5-26,7$ & 44,1 & $39,9-48,5$ & 14,7 & $11,3-18,9$ & 19,5 & $15,6-24,0$ & \\
\hline Frango & & & & & & & & & 0,187 \\
\hline Urbano & 20,6 & $17,3-24,3$ & 59,0 & $55,4-62,5$ & 13,9 & $11,8-16,3$ & 6,5 & $5,0-8,4$ & \\
\hline Rural & 27,2 & $23,2-31,6$ & 56,0 & $49,6-62,2$ & 12,2 & $9,3-16,0$ & 4,5 & $2,0-10,0$ & \\
\hline Peixe & & & & & & & & & $<0,001$ \\
\hline Urbano & 59,7 & $56,1-63,2$ & 50,8 & $46,0-55,7$ & 3,3 & $2,4-4,4$ & 1,0 & $0,5-1,7$ & \\
\hline Rural & 36,0 & $32,6-39,7$ & 38,6 & $34,3-43,1$ & 6,6 & $4,5-9,6$ & 4,0 & $2,3-6,7$ & \\
\hline Suco natural de frutas & & & & & & & & & 0,554 \\
\hline Urbano & 24,0 & $20,9-27,4$ & 29,3 & $26,4-32,4$ & 13,4 & $11,5-15,6$ & 33,3 & $30,1-36,6$ & \\
\hline Rural & 25,9 & $20,6-31,9$ & 32,0 & $27,4-37,0$ & 13,1 & $10,0-17,0$ & 29,1 & $23,6-35,2$ & \\
\hline Alimentos fritos & & & & & & & & & 0,059 \\
\hline Urbano & 47,4 & $43,4-51,5$ & 40,4 & $36,6-44,4$ & 6,5 & $5,1-8,3$ & 5,6 & $4,1-7,6$ & \\
\hline Rural & 56,1 & $50,3-61,7$ & 34,9 & $29,0-41,3$ & 4,2 & $3,0-5,9$ & 4,8 & $2,9-7,9$ & \\
\hline Doces & & & & & & & & & 0,003 \\
\hline Urbano & 26,7 & $23,8-29,9$ & 38,2 & $34,4-42,0$ & 12,0 & $10,0-14,4$ & 23,1 & $19,9-26,6$ & \\
\hline Rural & 36,4 & $31,5-41,7$ & 36,3 & $32,2-40,7$ & 12,9 & $9,5-17,2$ & 14,4 & $10,2-19,8$ & \\
\hline Biscoitos § & & & & & & & & & 0,026 \\
\hline Urbano & 7,7 & $6,1-9,6$ & 26,8 & $23,0-31,0$ & 17,7 & $15,1-20,7$ & 47,8 & $43,1-52,5$ & \\
\hline Rural & 13,0 & $10,0-16,8$ & 26,6 & $22,6-31,1$ & 20,1 & $16,1-24,9$ & 40,2 & $33,2-47,6$ & \\
\hline Salgadinhos §§ & & & & & & & & & 0,322 \\
\hline Urbano & 44,7 & $41,3-48,1$ & 38,8 & $35,7-42,1$ & 7,4 & $5,7-9,6$ & 9,1 & $7,2-11,4$ & \\
\hline Rural & 44,8 & $39,7-50,0$ & 41,8 & $36,5-47,3$ & 7,4 & $5,2-10,4$ & 6,0 & $4,0-8,8$ & \\
\hline Refrigerante §§§ & & & & & & & & & $<0,001$ \\
\hline Urbano & 24,1 & $21,5-26,9$ & 40,2 & $36,2-44,3$ & 10,7 & $8,7-13,1$ & 25,0 & $22,0-28,4$ & \\
\hline Rural & 35,5 & $30,9-40,5$ & 41,8 & $36,4-47,5$ & 12,9 & $9,5-17,2$ & 9,7 & $7,5-12,5$ & \\
\hline logurte & & & & & & & & & $<0,001$ \\
\hline Urbano & 20,6 & $18,0-23,3$ & 34,8 & $32,0-37,7$ & 15,9 & $12,7-19,8$ & 28,8 & $25,7-32,0$ & \\
\hline Rural & 40,2 & $34,1-46,7$ & 35,6 & $30,9-40,6$ & 11,6 & $8,1-16,4$ & 12,5 & $8,1-19,0$ & \\
\hline
\end{tabular}

IC95\%: intervalo de 95\% de confiança.

* $\mathrm{n}$ amostral sem expansão $=4.322$;

** Arroz e macarrão;

*** Batata, mandioca, mandioquinha (batata-baroa), cará, inhame, abóbora;

\# Feijões e lentilha;

\#\# Tomate, cenoura, beterraba, berinjela, chuchu e abobrinha;

\#\#\# Carne bovina e suína;

$\S$ Biscoitos e bolachas;

$\S \S$ Salgadinhos de pacote;

$\S \S$ Refrigerantes e sucos artificiais.

\section{Discussão}

Pela primeira vez no país, os dados oriundos da PNDS de 2006/2007 permitiram conhecer o consumo alimentar das crianças brasileiras com idade de 6-59 meses. Observou-se que a base da alimentação destas foi representada pelo consumo diário de arroz com feijão. Verificou-se, também, que a maioria não consumiu diariamente verduras de folhas, legumes, carnes e frutas, mas consumiu, na frequência diária ou de uma a três vezes na semana, biscoitos, refrigerantes, doces e salgadinhos 13 .

As diretrizes estabelecidas no país sobre alimentação saudável para crianças 20 apontam para a necessidade do consumo diário de frutas, verduras de folhas, legumes, leguminosas e carnes e a necessidade de se evitar o consumo 
de refrigerantes, biscoitos, doces, salgadinhos e outros alimentos não recomendados nos primeiros anos de vida das crianças. Por isso, é possível afirmar que o atual consumo alimentar das crianças brasileiras menores de cinco anos está muito aquém do preconizado.

A II Pesquisa Nacional de Prevalência de Aleitamento Materno 15 investigou práticas alimentares de crianças de até 12 meses e também trouxe dados preocupantes sobre o consumo de alimentos não recomendados. No dia anterior à investigação, 8,7\% das crianças de 9-12 meses consumiram café; $11,6 \%$, refrigerante; $71,7 \%$, biscoitos/salgadinhos. Os resultados referentes ao consumo de alimentos recomendados e não recomendados observados nesses estudos são preocupantes, uma vez que o consumo alimentar implica impacto imediato nas condições de saúde e nutrição das crianças no momento atual e ao longo de toda a vida.

No âmbito da saúde pública, tais resultados são alarmantes, pois o consumo de alimentos não recomendados poderia causar (e causa) as deficiências de micronutrientes e a elevada e crescente prevalência de excesso de peso em todas as idades. De acordo com a própria PNDS, $7,4 \%$ das crianças apresentavam excesso de peso, prevalência superior à observada para baixa estatura e baixo peso. Aproximadamente, um quarto das crianças apresentava anemia e hipovitaminose A, concomitantes ou de forma isolada ${ }^{9}$.

Os resultados apresentados neste estudo podem direcionar ações e políticas públicas, já que esses dados confirmam a heterogeneidade do país, também, em relação ao consumo alimentar. Todos os alimentos investigados mostraram variação significativa de acordo com a região de residência. De maneira geral, é possível observar que as crianças residentes nas regiões Sul, Sudeste e Centro-oeste consumiram com mais frequência os alimentos recomendados (arroz, pão, batata, feijão, verdura de folha, legumes, carne, iogurte), quando comparadas às crianças residentes no Norte e Nordeste. A exceção é para o consumo de frango, frutas e suco de frutas, que também foi elevado entre as crianças residentes na Região Nordeste, e para o consumo de peixe pelas crianças residentes no Norte.

Apesar de alguns avanços, as Regiões Norte e Nordeste do país ainda concentram o maior número de municípios com elevadas prevalências de insegurança alimentar grave, em comparação com as Regiões Sul e Sudeste; estas apresentam a maior parte de seus municípios com baixas e médias exposições à insegurança alimentar, o que poderia explicar os resultados observados neste estudo 22 . A baixa ingestão de frutas, verduras de folhas, legumes e carnes se repercute na baixa ingestão de micronutrientes e, por consequência, nas deficiências nutricionais. Estudo realizado na Região Norte, no Município de Acrelândia, no Acre, investigou as condições de saúde e nutrição de crianças com idade entre 6-24 meses e confirmou que a dieta ingerida por estas não supria as necessidades diárias de ácido fólico, vitamina $\mathrm{C}$, vitamina A, zinco e ferro, conforme recomendadas para essa faixa etária 23 . Apesar da qualidade da dieta das crianças residentes nas regiões Norte e Nordeste estarem muito aquém do recomendado, estudo demonstrou que a desnutrição infantil na Região Nordeste reduziu em cerca de $50 \%$, no período compreendido entre 1996 e 2006 (13,5\% para $6,8 \%$ ). Dois terços dessa redução foram atribuídos a quatro fatores: aumento da escolaridade materna; crescimento do poder aquisitivo das famílias; expansão da assistência à saúde e a melhoria nas condições de saneamento 24 .

Também, de maneira geral, é possível observar que as crianças residentes nas Regiões Sul e Sudeste consumiram, com mais frequência, os alimentos não recomendados (doces e refrigerantes), com exceção para o consumo de biscoitos, salgadinhos e alimentos fritos, que também foi elevado entre as crianças residentes no Nordeste. As crianças que vivem no Centro-oeste apresentaram frequências menores de consumo de alimentos não recomendados, quando comparadas às crianças do Sul e Sudeste. Essas três últimas regiões são reconhecidas como as mais desenvolvidas do país, o que justificaria o maior acesso a todos os alimentos investigados neste estudo. O rendimento domiciliar médio observado para as três é superior ao que se verifica para os domicílios do Norte e Nordeste. Nas áreas rurais e urbanas destas duas regiões, o valor médio do rendimento domiciliar foi significativamente menor do que os maiores valores (Sudeste, Sul e Centro-oeste) 9 .

No que se refere ao consumo alimentar observado nas crianças residentes na Região Nordeste, a maioria dos alimentos recomendados tem menor participação, no entanto é importante a participação de alimentos não recomendados. Esse fato poderia ser justificado por evidências que apontam que o consumo de alimentos altamente energéticos seria mais frequente em populações de baixa condição socioeconômica, devido ao seu baixo custo em comparação com alimentos de alta densidade de nutrientes 25,26.

Dentre as várias explicações para essas disparidades, a desigualdade social instalada no Brasil é o fator que mais claramente justifica essa situação. As famílias/ou cuidadores têm papel importante na determinação dos alimentos que as crianças menores de cinco anos consomem, 
no entanto grande parte das famílias brasileiras residem em domicílios com insegurança alimentar moderada ou grave 18 .

Os resultados das análises por zona de residência, urbana e rural, mostraram que as crianças que moram em área urbana têm mais acesso aos alimentos recomendados e não recomendados, quando comparadas às residentes em estrato rural. Estudo anterior, que avaliou a disponibilidade familiar de alimentos, também mostrou a maior participação na dieta de frutas, verduras de folhas, legumes e carne no meio urbano, enquanto, no meio rural, foi maior a participação de feijões e outras leguminosas de raízes e tubérculos. O estudo aponta o rendimento familiar como um importante determinante do acesso a esses alimentos e mostra que o aumento no rendimento familiar determina o aumento da participação na dieta de carnes, verduras de folhas e legumes, refeições prontas, pães, biscoitos e refrigerantes, ao passo que a diminuição da participação ocorre com feijões, arroz, raízes, tubérculos e açúcares 27. Igualmente, já foi demonstrado que a redução no preço de frutas, legumes e verduras, possível de ser obtida por meio de políticas públicas, poderia aumentar a participação desses alimentos na dieta nos domicílios 28 .

Para garantir o acesso à alimentação saudável, são necessárias amplas ações intersetoriais. No âmbito da saúde, o profissional de saúde pode, por meio do aconselhamento nutricional, contribuir para melhorar o atual cenário de consumo alimentar por parte da população brasileira. Já foi demonstrado que o aconselhamento em aleitamento materno e em alimentação complementar promove mudanças no consumo alimentar, independentemente da condição socioeconômica 1,13,29. Esse profissional pode, ainda, ser o articulador e o fomentador de ações intersetoriais, nos territórios, para promoção da saúde e segurança alimentar e nutricional 30 .

\section{Conclusão}

O presente trabalho proporcionou conhecer o consumo alimentar entre crianças brasileiras na idade de 6-59 meses. Os resultados apontam para a baixa frequência de consumo de alimentos recomendados, importantes para o crescimento (como verduras de folhas, legumes, frutas e carnes), e elevada frequência no consumo de alimentos não recomendados (biscoitos, doces, refrigerantes e salgadinhos diariamente ou de uma a três vezes na semana).

Também foi possível observar diferenças regionais em relação ao consumo dos alimentos. As crianças residentes nas regiões Sul, Sudoeste e Centro-oeste consumiram com mais frequência os alimentos recomendados, mas também consumiram com mais frequência os alimentos não recomendados. As residentes na Região Norte apresentaram os menores percentuais de consumo dos alimentos recomendados e não recomendados; aquelas residentes na Região Nordeste, apesar de terem consumido em menor frequência os alimentos recomendados, consumiram em maior frequência frutas, biscoitos e salgadinhos. As crianças residentes no estrato urbano consumiram com mais frequência alimentos recomendados e não recomendados.

Como recomendações, as ações para garantir o acesso e a promoção de uma alimentação saudável devem centrar-se em todas as regiões brasileiras, todavia o Norte e o Nordeste devem ser priorizados. Crianças residentes na zona rural, em todo o país, também são mais vulneráveis. Ações que visem a desestimular o consumo de alimentos não saudáveis devem privilegiar todas as regiões do país, sendo mais urgentes no Sul, Sudeste, Nordeste e na zona urbana. 


\section{Resumo}

O objetivo foi avaliar o consumo alimentar em crianças brasileiras de 6-59 meses de idade, por região e zona de residência. Trata-se de estudo descritivo transversal com 4.322 crianças investigadas na Pesquisa Nacional de Demografia e Saúde (2006/2007). Observou-se baixo consumo diário de verduras (12,7\%), legumes $(21,8 \%)$, carnes $(24,6 \%)$ e elevado consumo de refrigerantes (40,5\%), alimentos fritos (39,4\%), salgadinhos (39,4\%), doces (37,8\%), na frequência de uma a três vezes na semana. Comparando-se as regiões, as crianças residentes no Sul, Sudeste e Centro-oeste consumiram com mais frequência arroz, pão, batata, feijão, verdura de folha, legumes e carne, mas também consumiram, mais frequentemente, alimentos não recomendados para a idade, como doces e refrigerantes. Crianças da zona rural apresentaram menor consumo dos alimentos recomendados para a idade e, também, dos não recomendados, quando comparadas às crianças da zona urbana. O consumo alimentar evidenciado neste estudo não está de acordo com recomendações de alimentação saudável para crianças.

Consumo de Alimentos; Promoção da Saúde; Ciências da Nutrição Infantil

\section{Colaboradores}

G. A. Bortolini contribuiu na concepção do estudo, análise dos dados e redação do manuscrito. M. B. Gubert e L. M. P. Santos colaboraram na concepção do estudo, orientação e revisão do manuscrito.

\section{Referências}

1. Vitolo MR, Bortolini GA, Feldens CA, Drachler ML. Impactos da implementação dos dez passos da alimentação saudável para crianças: ensaio de campo randomizado. Cad Saúde Pública 2005; 21:1448-57.

2. Walker SP, Wachs TD, Gardner JM, Lozoff B, Wasserman GA, Pollitt E, et al. Child development: risk factors for adverse outcomes in developing countries. Lancet 2007; 369:145-57.

3. Vitolo MR, Bortolini GA, Dal Bó Campagnolo P, Feldens CA. Effectiveness of a nutrition program in reducing symptoms of respiratory morbidity in children: a randomized field trial. Prev Med 2008; 47:384-8.
4. Black RE, Allen LH, Bhutta ZA, Caulfield LE, de Onis $\mathrm{M}$, Ezzati M, et al. Maternal and child undernutrition: global and regional exposures and health consequences. Lancet 2008; 371:243-60.

5. Dewey KG, Adu-Afarwuah S. Systematic review of the efficacy and effectiveness of complementary feeding interventions in developing countries. Matern Child Nutr 2008; 4 Suppl 1:24-85.

6. Arimond M, Ruel MT. Dietary diversity is associated with child nutritional status: evidence from 11 demographic and health surveys. J Nutr 2004; 134:2579-85. 
7. Ruel MT. Operationalizing dietary diversity: a review of measurement issues and research priorities. J Nutr 2003; 133(11 Suppl 2):3911S-26S.

8. Feldens CA, Giugliani ER, Vigo A, Vitolo MR. Early feeding practices and severe early childhood caries in four-year-old children from southern Brazil: a birth cohort study. Caries Res 2010; 44:445-52.

9. Ministério da Saúde/Centro Brasileiro de Análise e Planejamento. Pesquisa nacional de demografia e saúde da criança e da mulher - PNDS 2006: dimensões do processo reprodutivo e da saúde da criança. Brasília: Ministério da Saúde; 2009. (Série G. Estatística e Informação em Saúde).

10. Grantham-McGregor S, Cheung YB, Cueto S, Glewwe P, Richter L, Strupp B. Developmental potential in the first 5 years for children in developing countries. Lancet 2007; 369:60-70.

11. Garden FL, Marks GB, Almqvist C, Simpson JM, Webb KL. Infant and early childhood dietary predictors of overweight at age 8 years in the CAPS population. Eur J Clin Nutr 2011; 65:454-62.

12. Rolland-Cachera MF, Deheeger M, Maillot M, Bellisle F. Early adiposity rebound: causes and consequences for obesity in children and adults. Int J Obes (Lond) 2006; 30 Suppl 4:S11-7.

13. Vitolo MR, Rauber F, Campagnolo PD, Feldens CA, Hoffman DJ. Maternal dietary counseling in the first year of life is associated with a higher healthy eating index in childhood. J Nutr 2010; 140:2002-7.

14. Fall CH, Borja JB, Osmond C, Richter L, Bhargava SK, Martorell R, et al. Infant-feeding patterns and cardiovascular risk factors in young adulthood: data from five cohorts in low- and middle-income countries. Int J Epidemiol 2011; 40:47-62.

15. Departamento de Ações Programáticas e Estratégicas, Secretaria de Atenção à Saúde, Ministério da Saúde. II pesquisa de prevalência de aleitamento materno nas capitais brasileiras e Distrito Federal. Brasília: Ministério da Saúde; 2009.

16. Saldiva SR, Venancio SI, Gouveia AG, Castro AL, Escuder MM, Giugliani ER. Influência regional no consumo precoce de alimentos diferentes do leite materno em menores de seis meses residentes nas capitais brasileiras e Distrito Federal. Cad Saúde Pública 2011; 27:2253-62.

17. Engle PL, Fernald LC, Alderman H, Behrman J, O'Gara C, Yousafzai A, et al. Strategies for reducing inequalities and improving developmental outcomes for young children in low-income and middle-income countries. Lancet 2011; 378: 1339-53.

18. Cavenaghi S. Aspectos metodológicos e comparabilidade com pesquisas anteriores. In: Ministério da Saúde/Centro Brasileiro de Análise e Planejamento, organizadores. Pesquisa nacional de demografia e saúde da criança e da mulher - PNDS 2006: dimensões do processo reprodutivo e da saúde da criança. Brasília: Ministério da Saúde; 2009. p. 13-32. (Série G. Estatística e Informação em Saúde).
19. Departamento de Ciência e Tecnologia, Ministério da Saúde/Centro Brasileiro de Análise e Planejamento. Pesquisa Nacional de Demografia e Saúde da Criança e da Mulher: PNDS 2006. Questionário da mulher. Brasília: Centro Brasileiro de Análise e Planejamento/Departamento de Ciência e Tecnologia, Ministério da Saúde; 2008.

20. Departamento de Atenção Básica, Secretaria de Atenção à Saúde, Ministério da Saúde. Dez passos para uma alimentação saudável. Guia alimentar para menores de dois anos. Um guia para o profissional da saúde na atenção básica. Brasília: Ministério da Saúde; 2010.

21. Coordenação-Geral da Política de Alimentação e Nutrição, Secretaria de Atenção à Saúde, Ministério da Saúde. Guia Alimentar para a população brasileira: promovendo a alimentação saudável. Brasília: Ministério da Saúde; 2005. (Série A. Normas e Manuais Técnicos).

22. Gubert MB, Benicio MH, Santos LM. Estimativas de insegurança alimentar grave nos municípios brasileiros. Cad Saúde Pública 2010; 26:1595-605.

23. Garcia MT, Granado FS, Cardoso MA. Alimentação complementar e estado nutricional de crianças menores de dois anos atendidas no Programa Saúde da Família em Acrelândia, Acre, Amazônia Ocidental Brasileira. Cad Saúde Pública 2011; 27:305-16.

24. Lima AL, Silva AC, Konno SC, Conde WL, Benicio $\mathrm{MH}$, Monteiro CA. Causes of the accelerated decline in child undernutrition in Northeastern Brazil (1986-1996-2006). Rev Saúde Pública 2010; 44:17-27.

25. Drewnowski A, Specter SE. Poverty and obesity: the role of energy density and energy costs. Am J Clin Nutr 2004; 79:6-16.

26. Drewnowski A, Monsivais P, Maillot M, Darmon N. Low-energy-density diets are associated with higher diet quality and higher diet costs in French adults. J Am Diet Assoc 2007; 107:1028-32.

27. Levy-Costa RB, Sichieri R, Pontes NS, Monteiro CA. Household food availability in Brazil: distribution and trends (1974-2003). Rev Saúde Pública 2005; 39:530-40.

28. Claro RM, Carmo HC, Machado FM, Monteiro CA. Income, food prices, and participation of fruit and vegetables in the diet. Rev Saúde Pública 2007; 41:557-64.

29. Albernaz E, Araujo CL, Tomasi E, Mintem G, Giugliani E, Matijasevich A, et al. Influence of breastfeeding support on the tendencies of breastfeeding rates in the city of Pelotas (RS), Brazil, from 1982 to 2004. J Pediatr (Rio J.) 2008; 84:560-4.

30. Jaime PC, Silva ACF, Lima AMC, Bortolini GA. Ações de alimentação e nutrição na atenção básica: a experiência de organização no Governo Brasileiro. Rev Nutr 2011; 24:809-24.

Recebido em 14/Out/2011

Versão final reapresentada em 23/Mai/2012

Aprovado em 11/Jun/2012 